\title{
On the Connection of Gamma-Ray Bursts and X-Ray Flashes
}

\section{Jakub Řípa*}

formerly: National Taiwan University / LeCosPA

No.1, Sec.4, Roosevelt Road, Taipei 10617, Taiwan (R.O.C)

E-mail: ripa.jakub@gmail.com

\section{Attila Mészáros}

\section{Charles University,}

Faculty of Mathematics and Physics, Astronomical Institute,

V Holešovičkách 2, 18000 Prague 8, Czech Republic

E-mail: meszaros@cesnet.cz

Classification of gamma-ray bursts (GRBs) into groups has been intensively studied by various statistical tests since 1998. It has been suggested that next to the groups of short/hard and long/soft GRBs there could be another class of intermediate durations. For the Swift/BAT database Veres et al. 2010 (ApJ, 725, 1955) it was found that the intermediate-duration bursts might be related to X-ray flashes (XRFs). On the other hand, Řípa and Mészáros 2016 (Ap\&SS, 361, 370) and Řípa et al. 2012 (ApJ, 756, 44) found that the intermediate-duration GRBs in the RHESSI database are spectrally too hard to be given by XRFs. Also, in the BATSE database the intermediate-duration GRBs can be only partly populated by XRFs. The key ideas of the Řípa and Mészáros 2016 (Ap\&SS, 361, 370) article are summarized in this poster.

7th Fermi Symposium 2017

15-20 October 2017

Garmisch-Partenkirchen, Germany

\footnotetext{
* Speaker.
} 